\title{
THE RE-CREATION OF ARTIFICIAL SUN FROM ATOM FOR FUEL IN LABORATORY
}

\author{
A.VEERAPANDIYAN \\ Nehru Memorial College, Dept of Physics, Puthanampatti, Trichy-621007 Tamil Nadu India. \\ Email :mathscien@gmail.com
}

\begin{abstract}
The human need was increasing day by day for every materials entity in the surface of our earth but our existing planet did not have much more potential of energy resources or some other things within. Therefore, we are forced to go alternative energy resources. If then whatever have to be used today that has been gone away for excess of accessing resources of energy. In this situations we were try to catch the process of natural secrets and its functional creations and the process of recreation of resources and its methods would help to rectify the human needs this is not easy but this way will success in some times in future probably.

Towards marching the future like this way of life the human requirement of energy resources was raised more and more. In our present condition existing level of natural resources has disappear roughly it will be taking after 50 years. For the reason to think of alternative resources for energy productions was to overcome need of energy resources in future. Some understanding of natural secrets of energy productions was may be help to maintain the requirement of energy resources in the future.

The suitable physically controlled reaction of chemical actions will be providing enormous amount of energy. The chemical synthesis of atoms and its replication with the pattern protections for maintenance of suitable atmospheric conditions were give the renewable resources for ever. This was organized by the energy creation of matter for suitable physical parameter association and dissociation character of law of selection.
\end{abstract}

Key words: Recreation, Atoms in molecules, Natural Resources, Chemical Reactions, Physical Transformation

\section{INTRODUCTION}

The foundation of physics has providing the path of function of chemical understanding of nature. For only the energy range is to making the difference of the all state of matter in the space. The requirement of higher energy and lowest minimum energy with the controlling capacity of knowledge is to providing the re-creation of matter and maintenance in the universe.

\section{RESULT AND DISCUSSION}

In generally this world was filled by the matter and energy or associated form. The re-creation of matter it is possible but we need high energy resources for create the matter that reason to create the natural resources for our laboratory. But in this work was tried creation of sun mainly harvesting the energy to meet the human needs in future generations. We know that the sun was surrounded by the gaseous molecules in mono atomic gas and etc...
The mono atomic molecules were moving everywhere in the different layer of sphere of the sun. For the layers each and everyone represented by the states of sphere there are containing different size of atoms sit with in the states. The dynamical relation of states was based on the temperature $T$ and pressure $P$. These particles were not interacted one another and therefore they are not colliding each other and transforming energy isotropic everywhere in the sphere of states. The temperature gradients it would make the variation of density of state of matter with in the space.

Sun provide us force to run the materials objects in the universe in other hand there was consider as closed systems then only not loose the force of unity. It is telling that the energy is not destroying itself but then creating other form of matter. And also this was maintaining the energy-mass conservation in the entire volume of the space.

Energy would be measured by the way suitable interval of density of states within the atom or array of 
atoms in the space. The entire space was separated by the many number of cells $\delta x$ and the state of aggregation is depend on the momentum $\delta p$. The $\delta x$ and $\delta p$ has to give the energy of the state of the systems for measured in localized. If one can see and get the relations the transition of state is KT and this would be distributed in the space order of entropy ds. One can see gaseous level of density of state of matter is $\rho=K T \delta \mathrm{V}$.

The gaseous laws of distribution of particles and energies were equivalent to $\sum e^{-\beta E}$ and these energies were filled by the volume $V$. Then final density of state of matter of the gas is $\sum g_{i} e^{-\beta E}$ here $g_{i}$ is a volume. The space and particle is to construct the limit of energies of the matter. The space was reduced much the law of distributions of particles that one is varying much someone comparing the state of gas.

The state of transition was altering the space reduction of matter. The symmetry of the space or matter has been to decide the energy of the systems.

Energy density of gas distribution in the space is $\sum g_{i} e^{-\beta E}$ and this distribution statistically to match the $E=V\left(\frac{2 \pi m K T}{h^{2}}\right)^{3 / 2}$.

Then energy of the systems it has been observed by the dynamical relation of the particle in the space. And the energy it could be measured by the intersection of functional components or physical parameter in the space.

$\delta_{i j}=1$ or 0 the distribution function of particles in the space mathematically this is equivalent to the beta function $\int_{0}^{1} x^{i}(1-x)^{j} d x$.

Here $i$ and $j$ is order of energy and matter distribution in the space

$$
\begin{gathered}
\int_{0}^{1} x^{i}(1-x)^{j} d x=\beta(i, j) \\
\delta_{i j}=1
\end{gathered}
$$

Energy and mass is perpendicular to each other and making the driving force of matter in the space for these self interactions.

$$
\frac{\delta_{i j}}{\int_{0}^{1} x^{i}(1-x)^{j} d x}=\frac{i j}{(i j)^{n}}
$$

When the space is get reduced much to the original state of the positions at the same times the state of matter is to reaching the stable state. Then the systems are getting expansion then loose the power of unity but then maintaining the strength of dynamics of matter very weakly the force of interactions.

The energy distribution is holding the solid state of matter $E_{i}$ (Fermion $)=\frac{E}{a^{\beta E}+1}$. where the temperatures are getting reducing much the space is condensed much more to the original state of gaseous molecules. The finally the state matter for the energy distribution is $E_{i}($ Boson $)=\frac{E}{g^{\beta E-1}}$. And then further transition of gaseous molecule is $\sum g_{i} e^{-\beta E}$.

The distribution of state of matter solid, liquid and gas is creating simultaneously without loss of mass and energy distribution in the space. This cyclic action has making the energy creation of the matter. The suitable tuning of physical parameters is making the law of continuity of physical transformation in the space.

The total energy of the universe was still constant and the localized point of view the energy will get changing in different point in the space. Only the wave approaches to match the creation of ideal universe without loss of mass and energy of the system. Here, consider as the energy is an elastic thread and broken by the many pieces if need they will connected nicely. Where the system is order and disorder they will get the different configuration. The symmetry of configurations will decide to the energy of the systems and stability of matter.

In reality the matter was constituted by the wave form for only a single thread .when if it is need to break and open then after release and absorbs the energy. The particles were travelling on the surface of the thread. This thread was compressing and expanding the applied force $\mathrm{P}$ (Pressure) or T (Temperature).

If the system temperature was go higher the density of states of matter to go higher. Therefore, the expansion of states is to get expand and the volume of the matter is get reduced for the condition for creations. For the reason 
was higher temperature $(\mathrm{T})$ the interaction minimized therefore entire De Broglie wave function condensed in the single point in the space to the reduction of volume in the space. And the wave function phase difference which is to making the higher amount of radiation in the sun.

Possibility of order of energy and mass association the reduction of volume

$$
E=\frac{i j}{(i j)^{n}} \delta V
$$

The wave function difference would be mathematically equivalent to the $\frac{1}{2}+\sum_{k=1}^{n} \operatorname{Cos} k(t-x)=\frac{\operatorname{Sin}\left(\frac{(2 n+1) n}{2}\right)}{2 \operatorname{Sin}\left(\frac{u}{2}\right)}$ Fourier series.

The volume expansion of state is to reduce the temperature and pressure the condensed waves are enlarged then interacted very strongly. The strong interaction of the state of energy is mutually coupled and mutually induction one another for other. And the mutual interactions to drive the system never lost the law of dynamics. The induction of matter and this amplification preserved or replicated by the chemical actions in the inner surface of the sun.

The De-Broglie wave functions $\lambda_{t h}=\frac{h}{(2 \pi m K T)^{1 / 2}}$, and then energy of the gaseous molecule is $E=V\left(\frac{2 \pi m K T}{h^{2}}\right)^{3 / 2}$

The gaseous state molecules was minimized by the volume and reached the solid matter form then the DeBroglie wave has $\lambda=\frac{h}{p}$.

Then the solid state wave equations resemble to the Schrödinger time dependent wave equations this equation has take care of the transformation of the materials points in the space.

$$
\begin{gathered}
\mathrm{H} \psi=\mathrm{Ci} \mathrm{h} \frac{\partial \psi}{\partial x}+\mathrm{V} \psi \\
\text { 2í h } \frac{\partial \psi}{\partial T}=\mathrm{C} \mathrm{i} \mathrm{h} \frac{\partial \psi}{\partial x}+2 \mathrm{~V} \psi \\
\Delta(\mathrm{x}, \mathrm{p})=\frac{\hbar}{2 \pi}=\operatorname{i~h} \frac{\partial \psi}{\partial x}
\end{gathered}
$$

Then rewrite the Schrödinger wave equation $2 i$ $\mathrm{h}(r) \frac{\partial \psi}{\partial T}=\mathrm{C} \frac{n h}{2 \pi}+2 \mathrm{~V}(\mathrm{r}) \psi$

$$
\begin{aligned}
& \text { 2í h }(r) \frac{\partial \psi}{\partial T}=\mathrm{C} \frac{n h}{2 \pi}+2 \mathrm{~V}(\mathrm{r}) \psi \\
& \text { Then, 2i hoC } \frac{n h}{2 \pi}=C \frac{n h}{2 \pi}+2 V(r) \psi \\
& \text { 2i h }(\Delta(\mathrm{x})) \mathrm{C} \frac{n h}{2 \pi}-\mathrm{C} \frac{n h}{2 \pi}=2 \mathrm{~V}(\mathrm{r}) \Psi \\
& \text { C } \frac{n h}{2 \pi}(2 i ́ h((\Delta(x))-1)=2 V(r) \psi \\
& \frac{n h}{2 \pi}=\frac{2 \mathrm{~V}((\Delta(\mathrm{x})) \psi}{c(2 \operatorname{lih}((\Delta(\mathrm{x}))-1)} \\
& P_{x}=\frac{2 \mathrm{~V}((\Delta(\mathrm{x})) \Psi}{c(2 \mathrm{l} h((\Delta(\mathrm{x}))-1)}
\end{aligned}
$$

Solid state materials De-Broglie wave function is $\lambda$ in

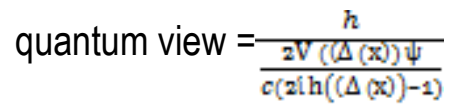

And then the expansion of entire universe density of state of matter is $\rho=\sum g T$. The macroscopic universe was bounded to the force of gravitation.

The distance of infinity to generating De-Broglie wave function is $\lambda=\frac{h}{p}$ and $E=\frac{h c}{\lambda}$.

Then long range force matter interactions and its structure of $\mathrm{E}=\left(\frac{1}{2} m \dot{r}^{2}+\frac{1}{2} m r^{2} \dot{\theta}^{2}+U(r)\right)$ substitute this value for De-Broglie waves then final result was provide the fixed point vibration of the particles in the plane in circular orbits or wave functions.

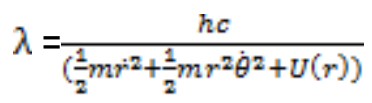

Then $h$ may be it will go Volume $(V), \lambda$ $=\frac{V(c)}{\left(\frac{1}{2} m \dot{r}^{2}+\frac{1}{2} m r^{2} \dot{\theta}^{2}+U(r)\right)}$

The $T$ and $P$ is one of the key components to create and destruct the materials entity in the universe. The function of $T$ and $P$ is to determine the properties of the matter and assembly of the fundamental entities of matter in the space.

If the order of atomic arrangements and this configurations it will give the energy state of the systems then the system energy changes to be observed by the law of entropy. 


$$
\mathrm{ds}=\left(\frac{d Q}{T}+K \ln W\right)
$$

$\mathrm{Kln} W$ Was give rise to the system configuration in the space and then change in configuration $K \ln W$ rise to the system heat $T d s$. The rise in heat is to do the work of the system likely the state of transformation of matter in the space

Overall change in entropy in mono atomic gas is $S=n R \log \left\{e^{5 / 2} \frac{V(2 \pi m K T)^{\mathrm{g} / 2}}{h^{\mathrm{s}}}\right\}$.

This final equation is to give the actual mechanism of the entropy rise in materials state of systems in varies states of association of configuration phase in the space.

\section{CONCLUSION}

This work was providing the way of understanding of the sun (system) chemical reactions collectively for varies associative actions. However, the understanding of collective effect of chemical actions and the physical processes were giving the condition of energy production of the nature and their maintenance with recreations.

\section{REFERENCE}

[1]. Goldstein, Poole, Safko. Classical Mechanics. Pearson Education, 2002.

[2]. Leonard. I. Schiff, Quantum Mechanics, Tata Mc Graw Hill Pvt Ltd. New Delhi.

[3]. L.D. Landaue and E.M. Lifshitz, Statistical physics. Institute of Physics Problem, Moscow.

[4]. Kerson Haung, Statistical Mechanics. John Wiley \&Sons, Inc, 1987.

[5]. Charles Kittel., Herbert Kroemes. Thermal physics, CBS Publishers \& Distributors. (1987).

[6]. Powell, J.L., and craseman, B., Quantum Mechanics, .Addison Wesley (1961).

[7]. Heat and Thermodynamics, Mark. W. Zemansky, McGrawHill Book Company, 1968. 